\title{
Development of a 3D Conceptual Design Environment Using a Head Mounted Display Virtual Reality System
}

\author{
Gabe Evans, Melynda Hoover, Eliot Winer \\ Iowa State University, Ames, Iowa, USA \\ Email: gabeevans2012@gmail.com,mthoover@iastate.edu, ewiner@iastate.edu
}

How to cite this paper: Evans, G., Hoover M. and Winer, E. (2020) Development of a 3D Conceptual Design Environment Using a Head Mounted Display Virtual Reality System. Journal of Software Engineering and Applications, 13, 258-277.

https://doi.org/10.4236/jsea.2020.1310017

Received: September 23, 2020

Accepted: October 19, 2020

Published: October 22, 2020

Copyright $\odot 2020$ by author(s) and Scientific Research Publishing Inc. This work is licensed under the Creative Commons Attribution International License (CC BY 4.0).

http://creativecommons.org/licenses/by/4.0/

\begin{abstract}
Engineering design is a resource intensive process with over half of a product's total cost being attributed to design stage decisions. Developing product designs often involves creating complex $3 \mathrm{D}$ models in a $2 \mathrm{D}$ environment, a non-trivial task. This paper details a proof of concept VR environment displayed in a commodity HMD, specifically the HTC Vive. The environment allows a user to create full-scale, 3D, product geometry at the conceptual phase of the design process. The conceptual design environment contains a World-in-Miniature (WIM) model for enhanced usability. This method allows a user to efficiently manipulate full-scale geometry by adjusting the corresponding parts on a WIM model. Free-form mesh deformation was also implemented to provide designers with flexibility and efficiency not found in traditional design packages. Vital design metrics (e.g., cost, weight, and center of mass) were incorporated to allow a user to perform preliminary design analysis to assess product feasibility. Throughout the development process, the unique challenges and affordances associated with commodity HMDs were identified, explored, and discussed in this paper. Ultimately, the VR application discussed in this paper allows the user to easily create, assess, and view conceptual 3D designs without the constraints of traditional CAD software.
\end{abstract}

\section{Keywords}

Virtual Reality, Computer Aided Design, Engineering Analysis

\section{Introduction}

Research suggests that as much as $50 \%-70 \%$ of a product's total cost can be attributed to design stage decisions [1] [2] [3]. Due to its substantial impact on the 
total cost and overall success or failure of a product, the engineering design process is a critical aspect of the product life cycle.

While many product design processes and paradigms exist, most can be broken down into four major stages: planning and task clarification, conceptual design, embodiment design, and detailed design [1]. The work presented in this paper focuses on the conceptual design portion of a product's lifecycle. Conceptual design involves generating numerous product concepts based on ideas, sketches, and product goals [4]. Therefore, it is extremely advantageous for a designer to have the right tools for the job.

Computer Aided Design (CAD) packages are the most common design too used during the conceptual design phase. However, for conceptual design, they lack key features [5] [6]. These features include immersive visualization, efficient geometry manipulation, freedom from mathematical constraints, and a simple interface [7] [8]. Additionally, CAD packages are known to suffer from feature bloat [5] [6], potentially hindering the software's usability, especially for novice designers. Additionally, CAD packages usually lack immersive visualization and the ability to directly manipulate complex geometry without knowing precise dimensions, among other requirements. As a result, a designer may spend an excessive amount of time comprehending, communicating, and implementing design changes.

Simple geometry manipulation tools that are unconstrained by mathematical relationships, the ability to import primitives and existing geometry, and preliminarily analysis features are all advantageous during conceptual design [7] [9] [10] [11]. If these tools are implemented properly, conceptual designers may be able to generate, iterate, and evaluate concepts much more efficiently than possible when using a CAD system. Therefore, simplified assessment tools can also reduce the amount of training necessary to use the software effectively.

While some software suited to conceptual design exist, none of them allow a user to simultaneously view and manipulate geometry in a commodity VR HMD. The recent arrival of commodity VR HMDs provides an opportunity to explore the potential time and cost saving benefits this technology may provide to designers [12] [13]. Hence, this work places a special focus on implementing conceptual design tools in a commodity VR HMD. Such a system could be used help designers understand and manipulate conceptual product designs.

To study this theory, a proof of concept application was developed to meld the benefits of commodity VR HMD technology with basic geometry manipulation and analysis tools. The work below details the development of the aforementioned system and a comparison of its features with a previous conceptual design tool, coupled with an immersive 3D viewer [10] [14]. Overall, the work presented in the paper is a step towards providing designers with an immersive conceptual design tool, displayed in a commodity HMD. Such a tool, unconstrained by CAD requirements, will hopefully aid a designer in the production of more robust designs while saving time and money. 


\section{Background}

The proof of concept system developed in this work relies on research contributions in both engineering design and VR technology. Those contributions guided the development of this work's conceptual design environment and interaction techniques within the commodity HMD. The previous scholarly contributions related are categorized into two main sections: 1) conceptual engineering design, 2) the benefits of VR for conceptual design, and 3) the tradeoffs of different VR hardware options.

\subsection{Conceptual Engineering Design}

The conceptual design process involves quickly generating product concepts by altering existing designs or developing new designs [1] [10]. Traditionally, conceptual design teams use CAD packages to conceptualize, design, visualize, and validate the feasibility of proposed designs. However, current CAD packages are not ideal for rapid prototyping because they are time consuming and difficult to use as a result of feature bloat and mathematical constraints [5] [6]. This prevents users from directly manipulating the model and stifles creativity in design. However, since many product designs are merely redesigns of existing geometry, the ability to manipulate existing geometry in an intuitive and efficient manner is highly desirable in a new prototyping tool, such as the one presented in this work [6] [12]. Another problem with existing CAD tools is learnability [15]. Reducing the complexity of a design package, by only implementing the tools necessary for conceptual design, would allow a novice designer to work more efficiently [10] [12] [15]. Based on the cited literature, it is clear that current CAD packages lack the flexibility needed for rapid prototyping design. Previous research by Noon et al. sought to fill this product gap by developing a conceptual design tool called the Advanced Systems Design Suite (ASDS). The tool is a conceptual design environment which allows a user to import and manipulate geometry with a toolset optimized for conceptual design [10]. The ASDS utilizes an immersive VR environment for concept visualization and to allow for the evaluation and assessment of designs. This environment was developed to bridge the gap between CAD and conceptual design [11] [16]. However, this tool did not allow the user to manipulate the model while immersed in the virtual environment. This work seeks to fill that gap and provide a fully immersive VR prototyping tool with model manipulation capabilities.

\subsection{Benefits of VR for Conceptual Design}

One way to enhance user understanding of the geometry is through immersive $3 \mathrm{D}$ visualization, such as in VR. In engineering design, the ability to fully understand a part and accurately interpret the displayed geometry is crucial [14] [17]. However, in their native format, design packages display 3D geometry on a $2 \mathrm{D}$ screen, making it difficult for a designer to quickly develop a full understanding of the geometry. Research suggests 3D immersive applications may allow users 
to more accurately form mental models and create more effective designs [18]-[24]. Additionally, the discrepancies that often arise between the mental models of individuals on a design team could result in extraneous time spent interpreting and understanding geometry, costing valuable resources [25] [26]. Using virtual reality to see designs in $3 \mathrm{D}$ can increase communication, successful task completion, and a better understanding of complex geometry for teams [21] [22] [25] [26].

VR allows a user to experience their design in environments which are not feasible in the real world because of cost, safety, or time. Eliminating these constraints by utilizing a Virtual Reality environment allows a team to better understand, explore, and evaluate otherwise impractical scenarios and alter designs earlier in the development process [27]-[32].

This ultimately helps design teams make better decisions in VR as compared to a traditional 2D design environment [15].

The aforementioned benefits of VR systems prompted research teams to explore the benefits of melding VR with design environments [10] [12]. Existing tools that use VR with CAD tools include Google SketchUp and Autodesk Revit. Google SketchUp is another package which aims to replace complex 3D modeling software with a more intuitive way of generating designs [33]. Google SketchUp allows users to draw in 3D and import simple primitives to explore concepts without being hindered by mathematical constraints. SketchUp Viewer for VR allows the user to look at their designs in immersive 3D environments. Although this plugin allows users to measure distances in VR, it lacks more detailed analysis tools that are important to solving engineering design problems. Autodesk Revit is a conceptual design environment that allows designers to explore ideas and perform preliminary analysis on their designs [34] [35]. While Autodesk Revit is considered a conceptual design environment, it is similar to CAD in the fact that a user must dimension each model, which takes considerable time and effort. Neither of these solutions allows the designer to manipulate model geometry in VR.

Similarly the ASDS, described earlier, allows designers to manipulate geometry in addition to assessing the cost, weight, and other key product parameters [16]. However, in the ASDS, geometry manipulation is done on a 2D desktop, and then viewed in a VR CAVE preventing the designer from editing their work while in VR. Switching between viewing environments takes an unnecessary amount of time and disrupts highly valuable user immersion, vital to understanding complex 3D geometry [19] [36]. The ability to simultaneously view and manipulate geometry in an immersive VR environment would potentially add value to the design process.

So far, there are no design solutions which provide the ability to easily manipulate $3 \mathrm{D}$ CAD models in VR without switching between a computer and an HMD. The decoupling of VR viewing systems from geometry manipulation tools is likely due to: 1) difficulties associated with model format conversions, 2) 
the high cost of a VR CAVE, and 3) the previous lack of commodity VR HMDs [12] [37] [38]. With the arrival of commodity VR HMDs, the latter two barriers are essentially eliminated. Consequently, this work seeks to evaluate the effectiveness and proposed benefits of fusing design tools with commodity HMDs.

\subsection{Tradeoffs of Different VR Hardware}

Commodity VR HMDs differ drastically from their CAVE ${ }^{\text {тा }}$ counterparts. An advantage of a CAVE ${ }^{\mathrm{sm}}$ is the ability for design teams to collaborate face to face without their view being fully occluded by a headset. However, a CAVE ${ }^{\text {Th }}$ requires an extensive amount of space, setup time, and cost while commodity HMDs do not. Commodity HMDs are powered by a single high-performance computer, while a $\mathrm{CAVE}^{\mathrm{m}}$ utilizes a cluster of high-performance machines. The low cost, minimal space, and high graphical fidelity of commodity HMDs allow them to be implemented into a design workflow more easily than bulky and costly CAVE ${ }^{\text {rw }}$ systems [39].

Commodity HMDs can be broken down into two categories: mobile and tethered. Mobile devices require a phone to provide the computing power and display. At the time of this paper, mobile HMDs do not provide suitable positional tracking or powerful 3D graphics capabilities. Tethered HMDs utilize external tracking sensors to provide 6-DOF tracking of a user's position and the position of controllers. Tethered HMDs rely on a computer to provide computational power. These systems, including the computer that powers them, are more expensive than mobile HMDs [40]. Therefore, due to their superior graphical fidelity, computing power, and tracking capabilities, tethered HMDs are more advantageous for conceptual design applications and were chosen for use in this research.

\section{Development}

This section details five major topics related to developing a VR system for conceptual design: the selection of VR hardware, user interface design, navigation in VR, geometry manipulation tools including WIM model manipulation, and assessment features. The following subsections will discuss how the application developed for this research serves the user in the design and assessment parts of the rapid prototyping process using a VR HMD. The conceptual framework of the designer's workflow is described in Figure 1.

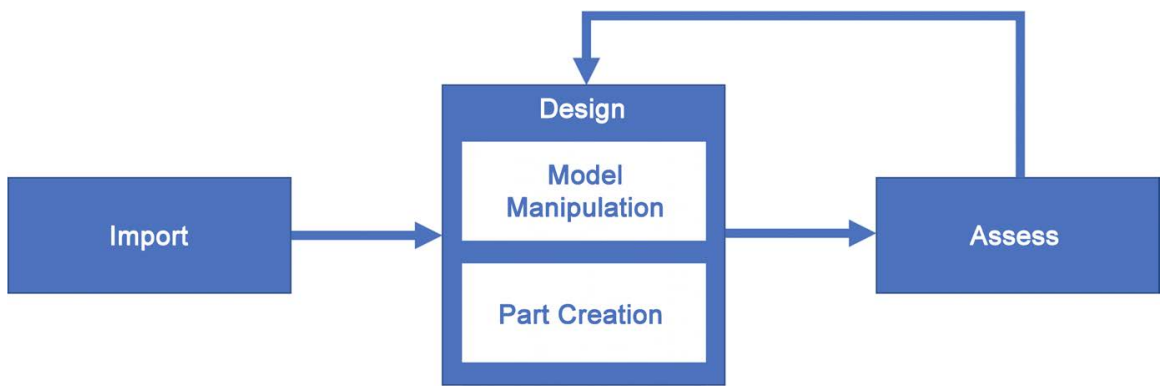

Figure 1. Conceptual Framework of the VR rapid prototyping process. 


\subsection{VR Hardware Selection}

The commodity VR headset chosen for this proof of concept environment was the HTC Vive. Factors contributing to the selection of the Vive were its controller and headset tracking precision, low cost, high resolution display, and controller functionality [40]. Tracking precision is very important due to its association with increased levels of immersion and an improved user experience, overall [12]. The Vive headset and two controllers are tracked in 6-DOF within a $3 \mathrm{~m}$ $\times 4 \mathrm{~m}$ tracked area, allowing for direct manipulation of virtual geometry [40]. The Vive controllers are equipped with buttons capable of detecting capacitive input from the user. User input is necessary to navigate menus and select the specific tools required for a variety of geometry manipulation operations. While other commodity HMDs, like the Oculus Rift and the HP Mixed Reality headset exist, the HTC Vive was chosen due to its capacitive touchpad and precise tracking in a larger area. The HTC Vive represents the tethered class of HMDs very well in terms of technical specifications and physical characteristics.

\subsection{User Interface Design}

Previous research found that interaction devices and virtual buttons must be easily accessible and intuitive [30]. In a CAVE ${ }^{\mathrm{mm}}$ a user can see interaction devices. However, in a fully occluded HMD a user cannot see the physical controller. Overlaying a model of the controller on the tracked controller provides a user with a visual representation of the controller's real-time position and orientation. Furthermore, a 3D overlay of buttons, mapped to virtual representations of the controllers, supplements a user's understanding of controller functionality.

The system created uses the capacitive touchpad to display dynamic buttons on the Vive controllers because previous research identified the advantages associated with recording a variety of user inputs via one interaction device [41] [42]. The displayed buttons change dynamically based on user input and the task at hand.

\subsection{Virtual Navigation}

While working with full-scale geometry of large products a user must be able to intuitively navigate about the environment to explore various vantage points. A virtual model of the right controller, with arrows overlaid on the touchpad, was utilized to aid a user with virtual navigation. Arrows dictate which direction a user will move when the button is depressed. Figure 2 shows the navigation controller and directional arrows.

A user's head orientation dictates the forward direction. Consequently, if a user holds down the forward arrow on the navigation touchpad, they will move in whichever direction their head is facing. Once a user navigates to their intended location, they may begin preforming geometry manipulation.

\subsection{Geometry Manipulation Tools}

In design environments, geometry manipulation is a necessary feature of a robust 


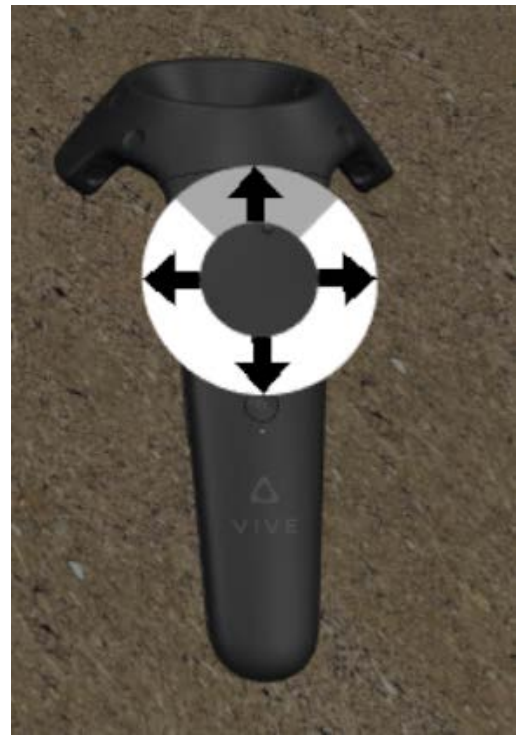

Figure 2. Navigation controller.

package. However, design environments must avoid incorporating an excessive number of features in order to deliver a system with a concise, intuitive, and highly usable interface. The implemented features include general part manipulation via translation, rotation, and scaling; WIM model manipulation; free-form deformation; and primitive import capabilities.

\subsubsection{General Part Manipulation}

A favorable conceptual design environment allows the user to efficiently and effectively manipulate existing geometry [5] [10]. Previous work on conceptual design environments has shown the importance of being able to translate, rotate, and scale objects in the environment [10] [42].

Within the HMD, geometry manipulation can be activated by pressing the desired button on the labeled part manipulation controller. The main menu, shown on the left in Figure 3 has buttons that allow the user to translate, rotate, scale, assess the geometry or display additional features relevant to the task at hand, as shown on the right of Figure 3. Currently, the geometry must be split into specific part groupings prior to importing the content into the VR environment because a hierarchical structure of all geometry is not displayed. For example, if a user wishes to move the front right tires of the combine they must group the rim, rubber tire, bolts, and all associated geometry as one part prior to utilizing the VR environment.

When using the translation, rotation, and scaling features a user must first select a part, intersect a controller with an axis on the transformation manipulator and press the trigger to grab the specific axis. The transformation manipulator is the three-axis component, shown in Figure 4, which allows a user to interact with geometry. This three-axis interface was chosen because it mimics the system used by many existing CAD software and uses existing mental models. It also allows the user to have more control over the deformation, translation, and 

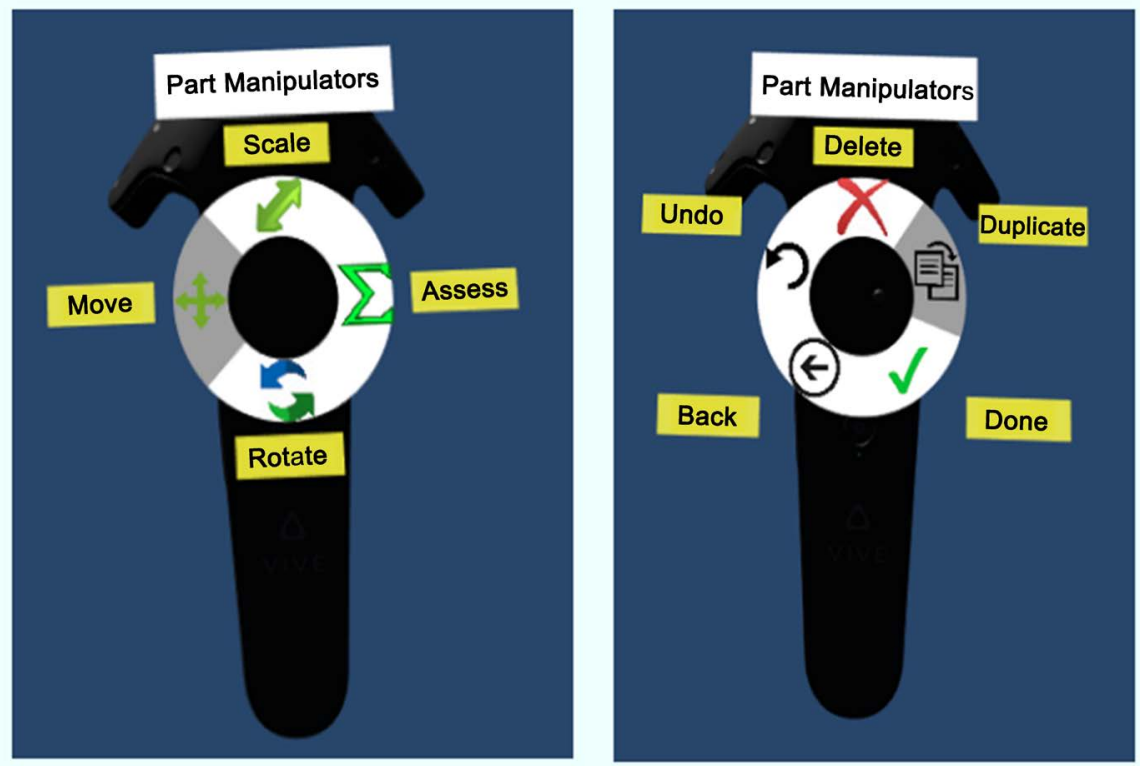

Figure 3. Part manipulation options.

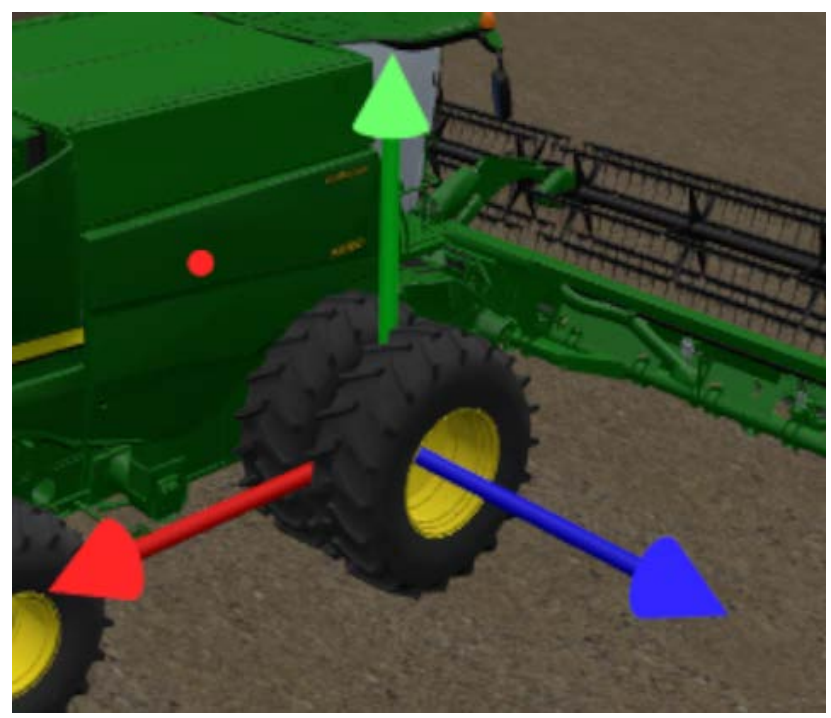

Figure 4. Transformation manipulator.

transformation along each axis because grabbing one axis of the transformation manipulator momentarily restricts geometry changes in the other two axes. Locking the movement of the parts to one axis at a time was implemented to provide the designer with added precision. Consider the geometry of a combine as shown in Figure 5.

A designer may want to examine visual and physical property changes that would arise from widening the wheelbase. Doing so would require the user to precisely move the tires outward along only one axis.

While manipulating parts in the proof of concept environment, the HMD's limited tracked space hindered the manipulation of full-scale geometry outside a user's normal range of motion. To address that issue, WIM model manipulation 


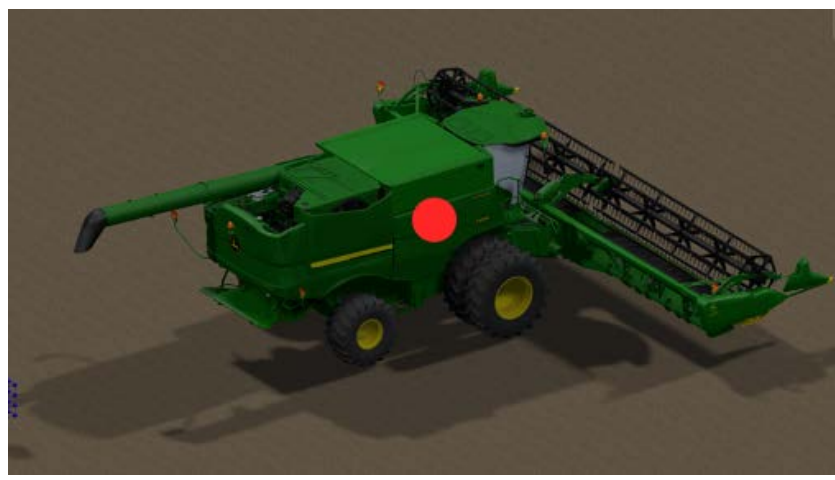

Figure 5. Combine geometry.

was explored.

\subsubsection{WIM Model Manipulation}

Visualization of full-scale geometry improves a user's understanding of the complex 3D geometry, aiding them in making well informed decisions [15]. The manipulation of full-scale geometry in VR is relatively easy when the geometry is small. However, ease of full-scale geometry manipulation does not translate to large scale assemblies such as industrial equipment or commercial airplanes. For example, if a user desired to move an airplane wing 15 feet they would have to select the virtual wing, move it a few feet, physically relocate to a new position, and repeat the process multiple times to achieve their goal. Repeating the process of selecting, translating, physically relocating, and deselecting is necessitated because the desired translation is outside the tracked volume of most commodity HMDs in addition to exceeding the capacity of a normal human range of motion. In the case of rotating a part, performing a 270-degree rotation about the vertical axis on a set of tires would require the user to grab the part and substantially reposition the wand, and their body, 270 degrees, a tedious task.

To overcome the difficulties which prevent efficient manipulation of large scale geometry, a WIM model of the large-scale geometry was implemented [43] [44]. The WIM model is essentially a duplicate of the full-scale geometry scaled down to a table top size so it can be easily manipulated by a user [45]. All component modifications on the WIM model are directly mapped to the full-scale assembly in a ratio consistent with the relationship between the scale of the small and large geometry. This mapping is illustrated in Figure 6 and Figure 7, before and after representations of the manipulated geometry. When a component on the WIM model is manipulated, a scaled transformation is applied to the corresponding component on the full-scale model.

Direct mapping aids a user in being able to perform coarse manipulations on the full-scale model through manipulations of the WIM model while preserving contextual information from the full-scale geometry. If a user is examining changes made to a full-scale combine, it is advantageous to be able to move those large parts via the WIM model. 


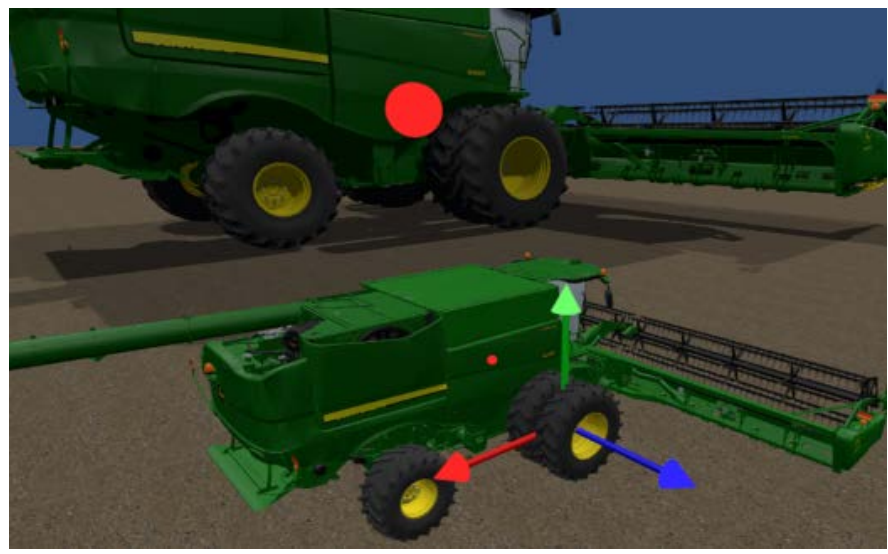

Figure 6. Direct mapping of geometry components-before.

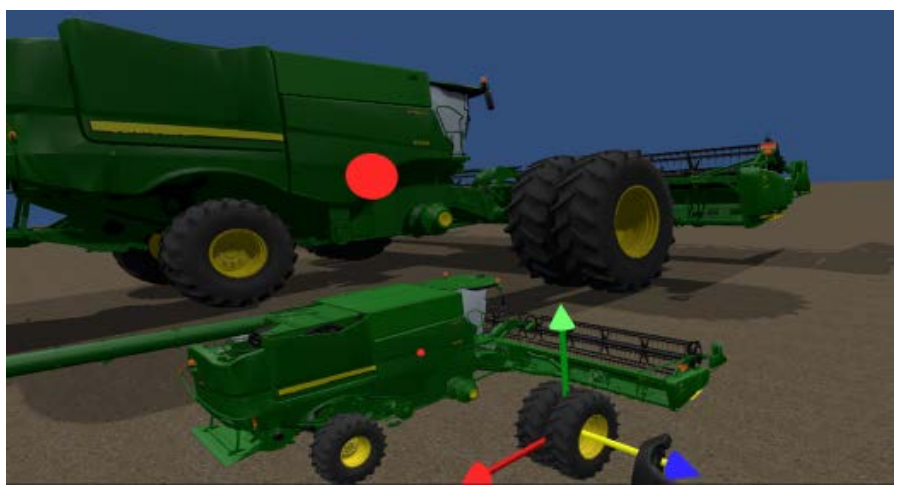

Figure 7. Direct mapping of geometry components-after.

Applying coarse modifications to the WIM model allows a user to quickly make gross adjustments to the full-size geometry assembly. However, there are limitations associated with utilizing the WIM method [45]. For example, WIM model manipulation is not very effective for making adjustments to geometry of drastically different scale factors. If a user wishes to manipulate the existing geometry on a more precise level, they should interact directly with the full-scale components.

In order for the WIM model to be an effective geometry manipulation aid, it must be easily accessible. Once the user reaches their desired position, they may press the "grip" button on the right controller to snap the WIM model to their location. Holding in the grip button on the right controller allows the user to move the WIM model. With the grip button depressed the position of the WIM model is identical to the right controller's position. However, the orientation of the WIM model stays aligned with the orientation of the full-scale geometry. Locking WIM model orientation maintains the mapping between the WIM model and the full-scale geometry. Once a user has placed the WIM model in a position that is advantageous for the task at hand, they may begin manipulating parts on the WIM model. When manipulating parts on the WIM model, a user will simultaneously see the adjustment being applied to the corresponding part on the full-scale assembly. 


\subsubsection{Free-Form Deformation}

Throughout the design process a user may wish to modify the shape of existing pieces of geometry. In many cases, a designer may wish to implement geometry modifications with a level of precision greater than simply scaling the part. A proven technique utilized to modify existing geometry is Free-form Deformation (FFD) [46] [47]. This technique involves a user moving control points surrounding a model in order to affect changes to the model. In this application, a user first selects the part they wish to modify. Upon part selection, a control box appears around the part. The size of the control box is set dynamically based on the size of the part's mesh. To provide precision without overcrowding the model the control box has 24 control points, scaled based on the size of the model. Connecting rods link the control points to provide the user with a sense of depth and a better understanding of which control points are connected to each other. Each control point is mapped to multiple vertices on the selected part. Bernstein Polynomials determine the relationship between vertices and control points [48]. A user may use either controller to grab a control point and freely drag it to a desired location. As control points are being repositioned, the part's mesh updates in real time based on the position of the control points. Real time mesh manipulation updates provide a user with immediate feedback based on the changes they are making. Figure 8 shows geometry prior to FFD manipulation while Figure 9 shows the same geometry after FFD manipulations have been applied.

Manipulated meshes in the virtual environment can be saved during runtime and exported in an OBJ file format for later viewing. Saving out altered meshes allows users to make free form changes to existing geometry then export a model

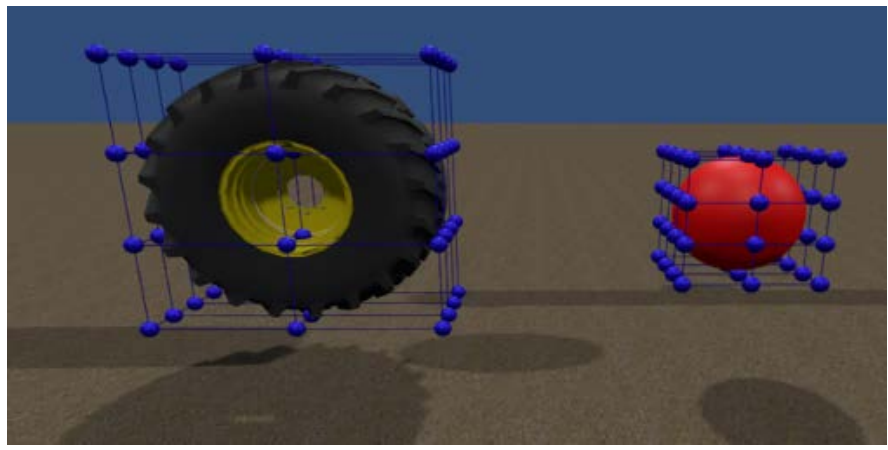

Figure 8. Before FFD manipulation.

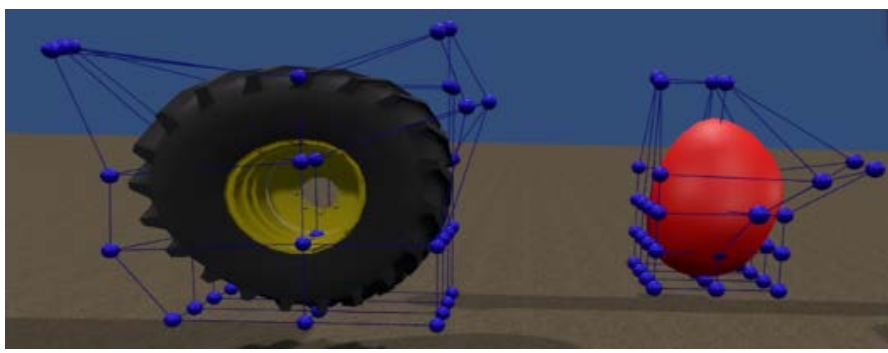

Figure 9. After FFD manipulation. 
of the adjusted part. Exporting the manipulated parts allows for later viewing, discussion, analysis, or potential recreation in a CAD package.

\subsection{Importing Primitives}

In addition to being able to freely deform existing geometry, certain situations benefit from allowing a user to insert primitives into their design environment. Case and user studies have also shown that being able to add primitives to an environment is beneficial to conceptual designers [49] [50]. Furthermore, being able to manipulate the primitives and existing geometry in a conceptual design environment is required [9] [12] [51].

After inserting basic primitives of 3D geometry, a user may scale the parts accordingly or use FFD to create entirely new geometry. To insert a primitive, a user simply selects the "primitives" button on their part manipulation controller and chooses from a menu of primitives including a cube, sphere, cylinder, and plane. Inserting primitives into the environment allows a user to represent new features or geometry.

\subsection{Assessment Tools}

Once a designer has applied desired changes to existing geometry, they may wish to evaluate their new design. Preliminary analysis tools were incorporated into this environment to allow for the evaluation of design feasibility. The ability to evaluate cost, mass, and weight distribution were all incorporated because they can be used to evaluate preliminary design feasibility. Additionally, collision detection between parts, and simple physics calculations, were implemented to ensure the physical realism of conceptualized designs. The summation of part costs and weight is displayed on an assessment pane which appears above the WIM model, when activated, as shown in Figure 10.

The tipping angle and center of mass of a vehicle may be evaluated by setting support points on tires or contact points. This allows a user to examine how adjusting weight distributions effects a product's center of mass. Figure 11 shows red and yellow support points, displayed as arrows, under the two visible wheels.

\section{Evaluation Method}

To preliminarily analyze the effectiveness of the interaction techniques, part manipulation tools, and the display device utilized in this work, the construction of a simple part was performed. In a previous work, Noon et al. created a simple double bearing assembly in both the ASDS and Solidworks, a commonly used CAD package, in order to analyze tradeoffs in each environment [1]. During the comparison Noon noted the fundamental differences in the processes used to develop the part in the ASDS versus CAD. The ASDS allowed for the import and simple manipulation and rearrangement of primitives while the CAD process required the user to create sketches, perform extrusions, and extruded cuts [10]. The creation process in CAD was more detailed and precise but it was 


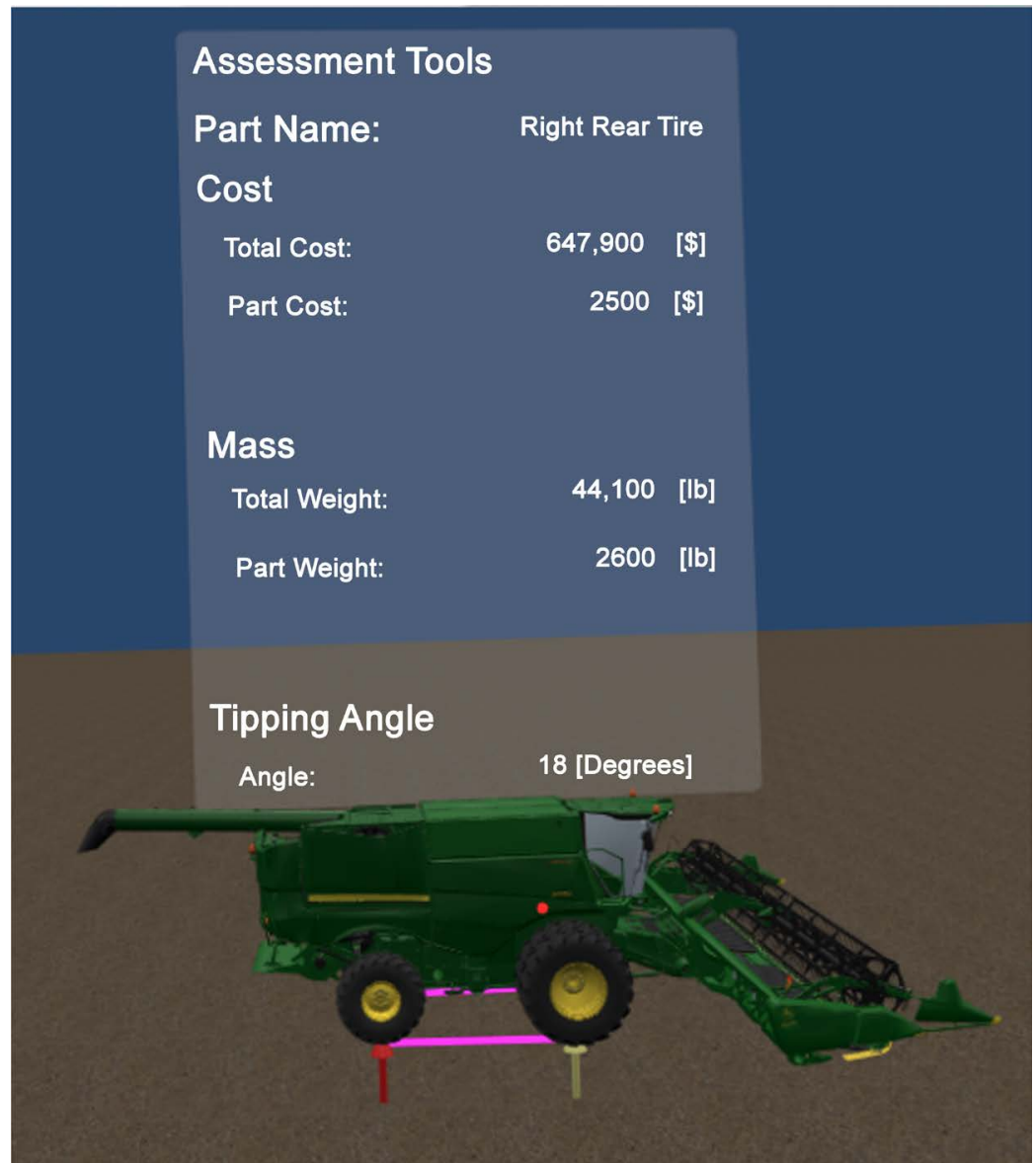

Figure 10. Assessment tools.

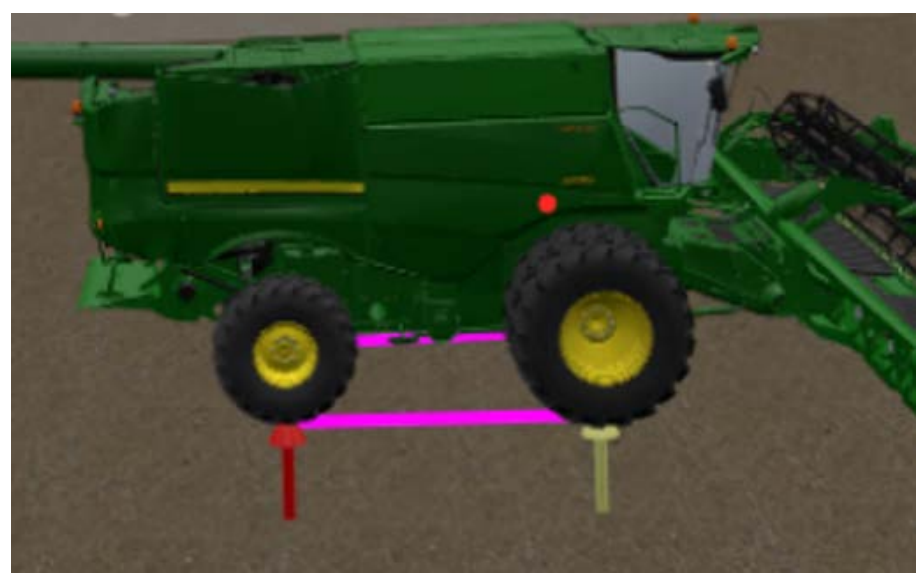

Figure 11. Support points on surface contact areas.

also much more timely.

Noon's ASDS package was utilized for the comparison with this system because the ASDS was found to contain most of the features identified in literature as necessary for effective conceptual design [11]. Noon et al. found the creation and modification of the test part was easier and more efficient in the ASDS than it was in the CAD package. While this work is not meant to compete with or re- 
place CAD systems, it was advantageous to compare the environment with the ASDS because the ASDS was found to be appropriate for the conceptual design stage [9] [15]. To maintain the ability to walk through the creation of each part, and qualitatively analyze the conceptual design environment described in this paper with the findings of Noon's work, the same part was created and altered. The purpose of generating the test part was to identify any advantages or pitfalls associated with this work's conceptual design environment displayed in a commodity HMD. While tradeoffs of each system were identified, it should be known that the proof of concept system in this work is not meant to be a replacement for CAD. The comparison was done simply to identify advantages of the conceptual design environment in a commodity VR system as well as areas for future work. The proof of concept VR environment is solely meant to be used as a tool to increase efficiency during conceptual design. An image of the part designed in both ASDS and this environment can be seen in Figure 12.

Creating the part in each design environment involved a very similar process. In this work's conceptual design environment, the front block shown in grey in Figure 12 was generated from a cube primitive, scaled appropriately. Next, a single blue pin was created by inserting a cylinder and rotating it accordingly. The additional pins were created by duplicating the original cylinder and translating them. The green block in the back of the part was generated by inserting another cube primitive and scaling it appropriately. The trapezoidal angle brackets on the back of the part were created by inserting a cube and utilizing a combination of scaling and FFD to create the angle on the bracket. Cylinders were inserted and scaled to represent the grey collars shown in the middle of Figure 12. The completed part in the current conceptual design environment is show in Figure 13.

\section{Results}

For a feasibility comparison, creation of this part was as simple as in the ASDS,

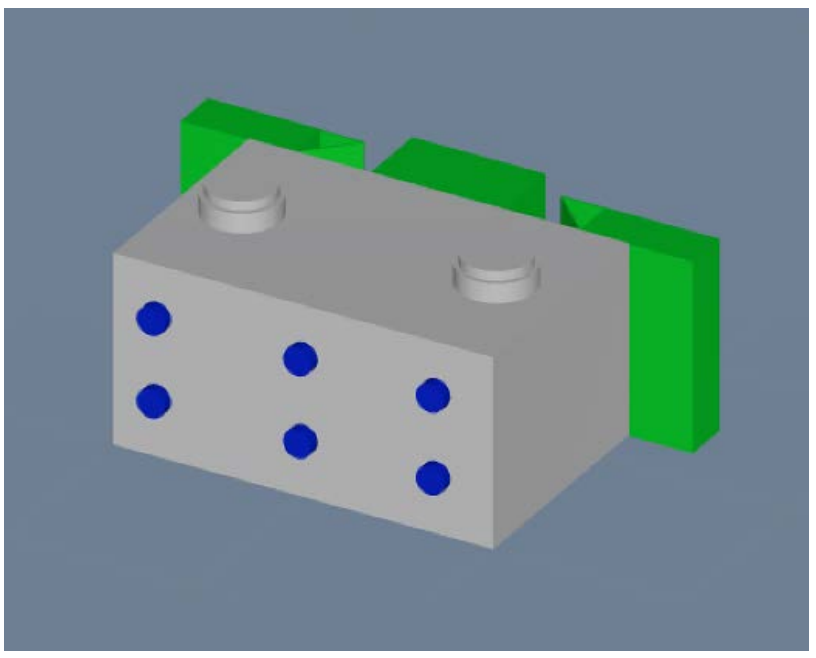

Figure 12. Example part created in the ASDS. 
and much easier than in a CAD package because it was not necessary to define feature dimensions explicitly. Creation and manipulation of geometry was efficient due to the ability to import primitives which represented certain aspects of the design. The primitives were then scaled to appropriate dimensions using tools which provided direct interaction with the geometry. With some additional refinements, a formal user evaluation could be conducted in the future. Table 1 , below, summarizes the qualitative advantages and disadvantages associated with the two systems. Overall the comparison was utilized to identify specific advantages and disadvantages associated with each system. Each system satisfied five of the seven parameters in this non-weighted comparison. The benefit of such a comparison is that pitfalls of each system are identified, and therefore can be improved upon in future work.

\section{Discussion}

During this comparison, it was apparent that a few refinements could be made to improve the overall user experience. When manipulating the angular bracket

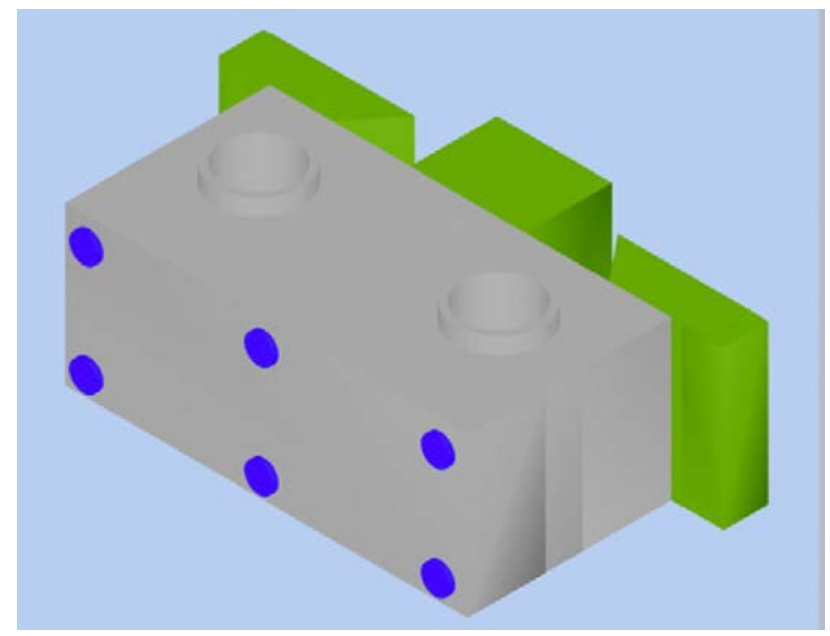

Figure 13. Test part in conceptual design VR App.

Table 1. Qualitative comparison between the ASDS and a conceptual design environment in a commodity.

\begin{tabular}{|c|c|c|}
\hline Parameter & $\begin{array}{l}\text { Advanced Systems } \\
\text { Design Suite (ASDS) }\end{array}$ & $\begin{array}{c}\text { Conceptual Design Environment } \\
\text { in a Commodity HMD }\end{array}$ \\
\hline Immersive Visualization & Yes & Yes \\
\hline $\begin{array}{c}\text { Manipulation in Immersive } \\
\text { Environment }\end{array}$ & No & Yes \\
\hline Extensive Primitive Library & Yes & No \\
\hline Appropriate Toolset & Yes & Yes \\
\hline Free-Form Deformation & No & Yes \\
\hline Ability to Import Geometry & Yes & Yes \\
\hline Component Multiselection & Yes & No \\
\hline
\end{tabular}


on the test part, control points along the edge of the bracket had to be adjusted individually. It would have been advantageous to be able to select multiple control points simultaneously so that only one translation of the control points was necessary. Being able to do so would improve precision and allow a designer the ability to make the angle uniform across each of the control points. Additionally, due to the limited number of primitives in the conceptual design environment, the collars had to be represented with cylinders. As shown in Figure 13, two cylinders are stacked on top of each other to visually represent the hollow collar. A more accurate representation of the collars would've been a cylindrical shape with the center removed. The more general issue here was a limited primitive library. Fortunately, adding primitives to the conceptual design environment is rather easy. Doing so simply requires generating the desired part in a CAD package and including it in the primitive's library.

Overall, the qualitative comparison of the test part generated in the ASDS and this work was fruitful. The test identified some minor pitfalls of this system, it's advantages, and areas for future work. The major advantages stemmed from the ability to simultaneously visualize and manipulate geometry in a fully tracked immersive environment, supporting the use of commodity HMDs. The test suggested that the application is on its way towards becoming a beneficial tool for VR conceptual design in addition to unveiling necessary future work.

\section{Conclusions}

The aforementioned proof of concept immersive design environment, displayed on a commodity VR HMD, takes a step towards the realization of conceptual design in commodity VR HMDs. Advances in technology have made commodity VR systems commercially available, allowing them to be an advantageous display for conceptual engineering design. The advantages of viewing, manipulating, and evaluating full scale models in a high-fidelity, immersive, 3D environment were qualitatively identified. An intuitive manipulation mechanism in the form of a WIM model and a natural 3D user interface allows users to harness the benefits of the 3D immersive environments found in VR.

The use of a low cost, commodity, HMD overcomes the hurdles associated with large and costly VR systems. The chosen HMD, the HTC Vive, also allows for collaborative applications to be built with multiple headsets so collaborative design and design review may be achieved.

Future work on this proof of concept system will incorporate three additional features: 1) the ability to import CAD models into a 3D VR environment in their native format, 2) the ability to select multiple parts or control points before performing transformations to provide more precision over the placement of components in the final design, 3) more primitive shape options to generate more realistic designs. Finally, a thorough evaluation in the form of a user study should be conducted on this proof of concept system and user feedback should be gathered to fine tune the user interface. 


\section{Conflicts of Interest}

The authors declare no conflicts of interest regarding the publication of this paper.

\section{References}

[1] Pahl, G., Beitz, W., Feldhusen, J. and Grote, K.H. (2007) Engineering Design: A Systematic Approach. Springer, Berlin. https://doi.org/10.1007/978-1-84628-319-2

[2] Al-Salka, M.A., Cartmell, M.P. and Hardy, S.J. (1998) A Framework for a Generalized Computer-Based Support Environment for Conceptual Engineering Design. Journal of Engineering Design, 9, 57-85. https://doi.org/10.1080/095448298261679

[3] Asiedu, Y. and Gu, P. (1998) Product Life Cycle Cost Analysis: State of the Art Review. International Journal of Production Research, 36, 883-908. https://doi.org/10.1080/002075498193444

[4] Wang, L., Shen, W., Xie, H., Neelamkavil, J. and Pardasani, A. (2002) Collaborative Conceptual Design-State of the Art and Future Trends. CAD Computer Aided Design, 34, 981-996. https://doi.org/10.1016/S0010-4485(01)00157-9

[5] Robertson, B.F. and Radcliffe, D.F. (2009) Impact of CAD Tools on Creative Problem Solving in Engineering Design. CAD Computer Aided Design, 41, 136-146. https://doi.org/10.1016/j.cad.2008.06.007

[6] Piegl, L.A. (2005) Ten Challenges in Computer-Aided Design. CAD Computer Aided Design, 37, 461-470. https://doi.org/10.1016/j.cad.2004.08.012

[7] Hsu, W. and Liu, B. (2000) Conceptual Design: Issues and Challenges. Computer-Aided Design, 32, 849-850. https://doi.org/10.1016/S0010-4485(00)00074-9

[8] Salzman, M.C., Dede, C., Loftin, R.B. and Chen, J. (1999) A Model for Understanding How Virtual Reality Aids Complex Conceptual Learning. Presence: Teleoperators and Virtual Environments, 8, 293-316. https://doi.org/10.1162/105474699566242

[9] Zboinska, M.A. (2015) Hybrid CAD/E Platform Supporting Exploratory Architectural Design. CAD Computer Aided Design, 59, 64-84. https://doi.org/10.1016/j.cad.2014.08.029

[10] Noon, C., Zhang, R., Winer, E., Oliver, J., Gilmore, B. and Duncan, J. (2012) A System for Rapid Creation and Assessment of Conceptual Large Vehicle Designs Using Immersive Virtual Reality. Computers in Industry, 63, 500-512.

https://doi.org/10.1016/j.compind.2012.02.003

[11] Bargar, A., Kulas, D., Poston, A., Richardson, T., Oren, M., Ph, D., Yilmaz, S., et al. (2011) Comparing ASDS to Existing Software For Engineering Conceptual Design, 3-8.

[12] Berta, J. (1999) Integrating VR and CAD. IEEE Computer Graphics and Applications, 19, 14-19. https://doi.org/10.1109/38.788793

[13] Havig, P., McIntire, J. and Geiselman, E. (2011) Virtual Reality in a Cave: Limitations and the Need for HMDs? Head- and Helmet-Mounted Displays XVI: Design and Applications, Vol. 8041, Article ID: 804107. https://doi.org/10.1117/12.883855

[14] Lau, H.Y.K., Mak, K.L. and Lu, M.T.H. (2003) A Virtual Design Platform for Interactive Product Design and Visualization. Journal of Materials Processing Technology, 139, 402-407. https://doi.org/10.1016/S0924-0136(03)00510-7

[15] Berg, L.P. and Vance, J.M. (2017) An Industry Case Study: Investigating Early Design Decision Making in Virtual Reality. Journal of Computing and Information 
Science in Engineering, 17, Article ID: 011001. https://doi.org/10.1115/1.4034267

[16] Renner, A., Thompson, F., Kalivarapu, V., Winer, E., Oliver, J., Gilmore, B. and Silver, D. (2015) An Application of Conceptual Design and Multidisciplinary Analysis Transitioning to Detailed Design Stages. 16th AIAA/ISSMO Multidisciplinary Analysis and Optimization Conference, Vol. 6, 1-13. https://doi.org/10.2514/6.2015-2647

[17] Strong, S. and Smith, R. (2001) Spatial Visualization: Fundamentals and Trends in Engineering Graphics. Journal of Industrial Technology, 18, 1-6.

[18] Rapp, D.N. (2005) Mental Models: Theoretical Issues for Visualizations in Science Education. In: Visualization in Science Education, Springer Netherlands, Dordrecht, 43-60. https://doi.org/10.1007/1-4020-3613-2 4

[19] Casas, L.A.A., Bridi, V.L. and Fialho, F.A.P. (1997) Virtual Reality Full Immersion Techniques for Enhancing Workers Performance. In: Re-Engineering for Sustainable Industrial Production, Springer, Berlin, 399-411. https://doi.org/10.1007/978-0-387-35086-8 34

[20] Broek, E., Meijer, F., Landman, R. and Broek, E. (2010) Augmenting Mental Models. CyberTherapy \& Rehabilitation, 3, 19-21.

[21] Buede, D.M. (2008) The Engineering Design of Systems: Models and Methods: Second Edition. Wiley, Hoboken.

[22] Boothroyd, G. (1994) Product Design for Manufacture and Assembly. Computer-Aided Design, 26, 505-520. https://doi.org/10.1016/0010-4485(94)90082-5

[23] Mathieu, J.E., Goodwin, G.F., Heffner, T.S., Salas, E. and Cannon-Bowers, J.A. (2000) The Influence of Shared Mental Models on Team Process and Performance. Journal of Applied Psychology, 85, 273-283. https://doi.org/10.1037/0021-9010.85.2.273

[24] Van Boven, L. and Thompson, L. (2003) A Look into the Mind of the Negotiator: Mental Models in Negotiation. Group Processes \& Intergroup Relations, 6, 387-404. https://doi.org/10.1177/13684302030064005

[25] Barton, J.A., Love, D.M. and Taylor, G.D. (2001) Design Determines 70\% of Cost? A Review of Implications for Design Evaluation. Journal of Engineering Design, 12, 47-58. https://doi.org/10.1080/09544820010031553

[26] Lyu, J., Chang, L.-Y., Cheng, C.-K. and Lin, C.-H. (2006) A Case Study Approach on the Development of Design Chain Operations Reference-Model in the Mold Industry. International Journal of Electronic Business Management, 4, 113-122.

[27] Tyagi, S. and Vadrevu, S. (2015) Immersive Virtual Reality to Vindicate the Application of Value Stream Mapping in an US-Based SME. International Journal of Advanced Manufacturing Technology, 81, 1259-1272.

https://doi.org/10.1007/s00170-015-7301-1

[28] Mujber, T.S., Szecsi, T. and Hashmi, M.S.J. (2004) Virtual Reality Applications in Manufacturing Process Simulation. Journal of Materials Processing Technology, 155-156, 1834-1838. https://doi.org/10.1016/j.jmatprotec.2004.04.401

[29] Choi, S.S., Kim, B.H. and Do Noh, S. (2015) A Diagnosis and Evaluation Method for Strategic Planning and Systematic Design of a Virtual Factory in Smart Manufacturing Systems. International Journal of Precision Engineering and Manufacturing, 16, 1107-1115. https://doi.org/10.1007/s12541-015-0143-9

[30] Bowman, D., Kruijff, E., LaViola Jr., J.J. and Poupyrev, I. (2004) 3D User Interfaces: Theory and Practice. CourseSmart eTextbook. https://books.google.com/books?hl=en\&lr=\&id=JYzmCkf7yNcC\&pgis=1

[31] Satter, K. and Butler, A. (2015) Competitive Usability Analysis of Immersive Virtual 
Environments in Engineering Design Review. Journal of Computing and Information Science in Engineering, 15, Article ID: 031001. https://doi.org/10.1115/1.4029750

[32] Bochenek, G.M. and Ragusa, J.M. (2004) Improving Integrated Project Team Interaction through Virtual (3d) Collaboration. Engineering Management Journal, 16, 3-12. https://doi.org/10.1080/10429247.2004.11415243

[33] Chopra, A. and Town, L. (2008) Introduction to Google SketchUp. Wiley Pathways, xxviii, 452 p., 16 p. of Plates.

https://www.google.com/books/edition/ /bP2oF0 Uc0YC?hl=en\&gbpv=0

[34] Zolotova, J., Vatin, N., Tuchkevich, E. and Rechinsky, A. (2015) Autodesk Revit-Key to Successful Training of Highly Qualified Civil Engineers. Applied Mechanics and Materials, 725-726, 1617-1625.

https://doi.org/10.4028/www.scientific.net/AMM.725-726.1617

[35] Nguyen, T.H., Shehab, T. and Gao, Z. (2010) Evaluating Sustainability of Architectural Designs Using Building Information Modeling. The Open Construction and Building Technology Journal, 4, 1-8. https://doi.org/10.2174/18748368010040100001

[36] Bowman, D. and McMahan, R.P. (2007) Virtual Reality: How Much Immersion Is Enough? Computer, 40, 36-43. https://doi.org/10.1109/MC.2007.257

[37] Kalivarapu, V., Macallister, A., Hoover, M., Sridhar, S., Schlueter, J., Civitate, A., Chernoff, G., et al. (2015) Game-Day Football Visualization Experience on Dissimilar Virtual Reality Platforms. Proceedings of SPIE - The International Society for Optical Engineering, Vol. 9392, 939202. https://doi.org/10.1117/12.2083250

[38] McMahan, R.P., Gorton, D., Gresock, J., McConnell, W. and Bowman, D.A. (2006) Separating the Effects of Level of Immersion and 3D Interaction Techniques. Proceedings of the ACM Symposium on Virtual Reality Software and Technology, Limassol, 1-3 November 2006, 108-111. https://doi.org/10.1145/1180495.1180518

[39] DeFanti, T.A., Dawe, G., Sandin, D.J., Schulze, J.P., Otto, P., Girado, J., Rao, R., et al. (2009) The StarCAVE, a Third-Generation CAVE and Virtual Reality OptIPortal. Future Generation Computer Systems, 25, 169-178. https://doi.org/10.1016/j.future.2008.07.015

[40] DigitalTrends (2019) Oculus Rift vs. HTC Vive. Spec Comparison. Digital Trends. Digital Trends Website. https://www.digitaltrends.com/virtual-reality/oculus-rift-vs-htc-vive

[41] Hotelling, I.S., Huppi, B.Q., Strickon, J.A., Kerr, D.R., Chaudhri, I., Christie, G. and Ive, J.P. (2017) Mode-Based Graphical User Interfaces for Touch Sensitive Input Devices. 1-5.

[42] Hand, C. (1997) A Survey of 3D Interaction Techniques. Computer Graphics Forum, 16, 269-281. https://doi.org/10.1111/1467-8659.00194

[43] Billinghurst, M., Baldis, S., Matheson, L. and Philips, M. (1997) 3D Palette. In: Proceedings of the ACM Symposium on Virtual Reality Software and Technology, ACM Press, New York, 155-156. https://doi.org/10.1145/261135.261163

[44] Pausch, R., Burnette, T., Brockway, D. and Weiblen, M.E. (1995) Navigation and Locomotion in Virtual Worlds via Flight into Hand-Held Miniatures. In: Proceedings of the ACM SIGGRAPH Conference on Computer Graphics, ACM Press, New York, 399-400. https://doi.org/10.1145/218380.218495

[45] Wingrave, C.A., Haciahmetoglu, Y. and Bowman, D.A. (2006) Overcoming World in Miniature Limitations by a Scaled and Scrolling WIM. 3DUI 2006: IEEE Sympo- 
sium on $3 D$ User Interfaces 2006 Proceedings, Vol. 2006, 11-16.

[46] Coquillart, S. (1990) Extended Free-Form Deformation: A Sculpturing Tool for 3D Geometric Modeling. ACM SIGGRAPH Computer Graphics, 24, 187-196.

https://doi.org/10.1145/97880.97900

[47] Sederberg, T.W. and Parry, S.R. (1986) Free-Form Deformation of Solid Geometric Models. Proceedings of the 13th Annual Conference on Computer Graphics and Interactive Techniques, Vol. 20, 151-160. https://doi.org/10.1145/15922.15903

[48] Gonzalez Hidalgo, M., Mir Torres, A. and Varona Gomez, J. (2013) Deformation Models: Tracking, Animation and Applications. Lecture Notes in Computational Vision and Biomechanics, Vol. 7, Springer, Berlin.

https://doi.org/10.1007/978-94-007-5446-1

[49] De Araújo, B.R., Casiez, G., Jorge, J.A. and Hachet, M. (2013) Mockup Builder: 3D Modeling on and above the Surface. Computers and Graphics, 37, 165-178. https://doi.org/10.1016/j.cag.2012.12.005

[50] Hsu, W. and Woon, I.M.Y. (1998) Current Research in the Conceptual Design of Mechanical Products. CAD Computer Aided Design, 30, 377-389.

https://doi.org/10.1016/S0010-4485(97)00101-2

[51] Khandani, S. (2005) Engineering Design Process: Education Transfer Plan. https://dphu.org/uploads/attachements/books/books 2547 0.pdf 